\title{
INSEPARABLE FINITE SOLVABLE GROUPS
}

BY

\author{
HOMER BECHTELL
}

\begin{abstract}
A finite group is called inseparable if the only proper normal subgroup over which it splits is the identity element. The $E$-residual, for the formation $E$ of groups in which all Sylow subgroups are elementary abelian, appears to control the action of splitting. In this article, inseparable solvable groups are identified that have a metacyclic Fitting subgroup and the $E$-residual a $p$-group.
\end{abstract}

1. Introduction. Attention is given to finite groups. The notation used is considered to be standard and may be found in [8] with the exception that $G=$ $[A] B$ denotes the semidirect product of the normal subgroup $A$ of $G$ with the subgroup $B$ of $G$. A reduced product $G$ of the normal subgroup $A$ by the subgroup $B$ is the product $G=A B$ such that $A C$ is a proper subgroup of $G$ for each proper subgroup $C$ of $B$. Frequently reference will be made to [8], because of its availability, rather than to the original article.

DEFINITION 1.1. A finite group is called inseparable if the only normal subgroups over which it splits are the identity element and the group itself.

It follows from the definition that all nontrivial simple groups and groups of one element are inseparable.

Motivation for this study stems from several directions. Each group $G$, $|G| \neq 1$, can be decomposed into the form $G=A_{n} A_{n-1} \cdots A_{1},\left|A_{i}\right| \neq 1$ for $i=1, \ldots, n,\left(A_{n} \cdots A_{j+1}\right) \triangleleft\left(A_{n} \cdots A_{j}\right),\left(A_{n} \cdots A_{j}\right)=\left[A_{n} \cdots A_{j+1}\right] A_{j}$ for $1 \leqslant j<n$, and $A_{i}$ is inseparable for $i=1, \ldots, n$. If $n \geqslant 2$, then $G$ is called a separable group. Consequently each group is formed through a succession of semidirect products by inseparable groups. The primary interest with such a decomposition is in regard to groups that are not expressible as a direct product. If a group $G$ is defined by an extension $1 \rightarrow N \rightarrow G \rightarrow A \rightarrow 1$, then it is only natural to examine $G$ to see if there exists a nonidentity proper normal subgroup over which $G$ splits and to express $G$ as a semidirect product. The extreme case occurs whenever $G$ does not split over any nonidentity proper normal subgroup, for example, the generalized quaternion group.

A classification of the inseparable $p$-groups would be an onerous task. The

Received by the editors July 24, 1973.

AMS (MOS) subject classifications (1970). Primary 20D40, 20D25, $20 \mathrm{D} 54$.

Key words and phrases. Finite solvable groups, inseparable groups, $p$-groups. 
obvious ones are the cyclic groups of prime power order, the generalized quaternion groups, and $p$-groups of maximal class that have all elements of order $p$ contained in the Frattini subgroup. In $\S 4$, the inseparable metacyclic $p$-groups are identified for $p>2$. For odd primes $p$, there is only one inseparable $p$-group of order $p^{4}$, and this group exists only for $p=3$; it has maximal class (see p. 334 of [8]).

Inseparable supersolvable groups are $p$-groups, as well as inseparable solvable $A$-groups and $p$-nilpotent groups. However solvable, but nonnilpotent, inseparable groups do exist as the next example indicates. Its uniqueness will be identified in Theorem 5.1.

EXAMPLE 1.2. Let $H=\left\langle a, b \mid a^{4}=1, b^{2}=a^{2}, b^{-1} a b=a^{3}\right\rangle$ be the quaternion group and $K=\left\langle x, y \mid x^{3}=y^{4}=1, x^{y}=x^{2}\right\rangle=[\langle x\rangle]\langle y\rangle$. There exists a homomorphism $\theta: K \rightarrow \operatorname{Aut}(H)$ having $\operatorname{Ker}(\theta)=\left\langle y^{2}\right\rangle$ and image isomorphic to the symmetric group of degree three. Form the semidirect product $G=[H] K$ with respect to $\theta$. The mapping $\gamma: a^{2} \rightarrow y^{2}$ is an isomorphism from $Z(H)$ onto $Z(K)$. Following the method of $D$. Gorenstein for forming partial semidirect products (p. 27 of [7]), set $D=\left\{z^{\gamma} z^{-1} \mid z \in Z(H)\right\}=\left\langle a^{2} y^{2}\right\rangle$. Consider $\bar{G}=$ $G / D$. Then $\bar{G}=\bar{H} \bar{K}$ for, $\bar{H}=H D / D \cong H, \bar{K}=K D / D \cong K, \bar{H} \triangleleft \bar{G}$, and $\bar{H} \cap \bar{K}$ $=Z(H) D / D \cong Z(H)$. A Sylow 2-subgroup of $\bar{G}$ is a generalized quaternion group of order $2^{4}$ and a Sylow 3-subgroup is cyclic.

Suppose that $\bar{G}=[\bar{N}] \bar{M},|\bar{N}| \neq 1$. Since a Sylow 3-subgroup is not normal in $\bar{G}$, then $|\bar{N}| \neq 3$ and 2 divides $|\bar{N}|$. Hence there exists a Sylow 2 -subgroup $\bar{P}$ such that $\bar{P}$ splits over $\bar{N} \cap \bar{P}$. But a generalized quaternion group does not split over any nonidentity proper normal subgroup. Hence $\bar{P} \subseteq \bar{N}$. Since $\bar{P} \pitchfork \bar{G}$, then 3 divides $|\bar{M}|$. Therefore $\bar{N}=\bar{G}$. So $\bar{G}$ is inseparable.

The study of inseparable groups inadvertently reflects on our knowledge of the structural components within a group that permit splitting over a normal subgroup. One of these components is the $E$-residual (or elementary commutator in [1]) for the formation $E$ of groups having all Sylow subgroups elementary abelian. Let $G_{E}$ denote this residual in a group $G$. It is known (see [1] , $[2$, p. 66]) to have the following properties:

(1.3) $G_{E}$ is generated by the Frattini subgroups of all Sylow $p$-subgroups of $G$.

(1.4) $G / G_{E}$ splits over each normal subgroup.

(1.5) If $G$ splits over $M=N \cap G_{E}$ for a subgroup $N \triangleleft G$, then $G$ splits over $N$.

The dihedral group indicates that the converse of (1.5) is not valid since in a nilpotent group, $G_{E}=\Phi(G)$.

(1.6) $G$ splits over each normal subgroup of $G$ if and only if $G$ splits over each normal subgroup of $G$ that is contained in $G_{E}$.

In the case of solvable groups, the property $F$ of splitting over each normal subgroup is a formation (see [3]). Denoting the $F$-residual of a group $G$ by $G_{F}$, 
it is noted that $1 \subseteq \Phi(G) \subseteq G_{F} \subseteq G_{E}$. Examples of groups exist for each of the possibilities that could occur for the various equalities and proper containments. In particular for $p$-groups, $G_{F}=\Phi(G)=G_{E}$. Groups satisfying (1.6) have $G_{F}$ $=1$.

As the symmetric group $S$ of degree four indicates, $S_{E}$ nilpotent does not imply that $S_{E}$ is either $S_{F}$ or $\Phi(S)$. In $S, \Phi(S)=S_{F}=1$, and $S_{E}$ is isomorphic to the elementary abelian normal subgroup of order four.

There are several reasons for the restrictions put on $G_{E}$ in this article. For control purposes, it seems reasonable to have the Frattini subgroup of a Sylow $p$-subgroup to be different than the identity element for only one prime. To allow $G_{E}$ to be nilpotent forces $G_{F}=\Phi(G)$ by Corollary 3.1. However, this still allows too much latitude on the nilpotency class of the Sylow $p$-subgroup for the prime $p$. For instance, C. Christensen [5] has given an example of a group in $F$ in which the Sylow $p$-subgroups can be of arbitrarily large nilpotence class. If the $E$-residual is a metacyclic $p$-group, then the corresponding Sylow $p$-subgroup of $G$ has a Frattini subgroup which is also metacyclic. There is enough known on the structure of these $p$-groups (see [4]) to be of use here.

In $\S 2$, general properties of inseparable groups related to the $E$-residual will be given. The remainder of the article will examine the case that the $E$ residual is a metacyclic $p$-group. $\S \S 3$ and 5 will deal with the inseparable group that is solvable but not a $p$-group and $\S 4$ is concerned with $p$-groups.

2. General properties. Epimorphic images, subgroups, and normal subgroups of inseparable groups are not necessarily inseparable. The cyclic groups of prime power order and the generalized quaternion groups raise a question on the type of group that has each subgroup inseparable.

THEOREM 2.1. A group $G$ has each subgroup $H \subseteq G$ inseparable if and only if $G$ is either a cyclic group of prime power order or a generalized quaternion group.

Proof. By induction on the order of $G$, each proper subgroup of $G$ is either a cyclic $p$-group or a generalized quaternion group. $G$ must be nilpotent for otherwise $G$ is a minimal nonnilpotent group and such groups split over some Sylow subgroup. Hence $G$ is a $p$-group and the result follows. The converse is evident in either case.

The next theorem answers several questions on the extreme cases involving epimorphic images of solvable groups.

THEOREM 2.2. (1) Each epimorphic image of a solvable group $G$ is inseparable if and only if $G$ is cyclic of prime-power order.

(2) $A$ solvable group $G$ is separable, but has each proper epimorphic image 
inseparable, if and only if $G$ satisfies one of the following for primes $p$ and $q$ :

(a) $G$ is elementary abelian of order $p^{2}$.

(b) $G=\left\langle x \mid x^{p}=1\right\rangle \otimes\left\langle y \mid y^{q}=1\right\rangle, p \neq q$.

(c) $G=[A] B$, for $B$ a cyclic p-group acting faithfully and irreducibly on the elementary abelian q-group $A$ and $p \neq q$.

Proof. Consider (1). Each abelian group satisfying this condition must be a cyclic $p$-group. So $G / G^{\prime}$ is a cyclic $p$-group. Suppose that $G^{\prime} \neq G^{\prime \prime}$. Then $G^{\prime} / G^{\prime \prime}$ contains a subgroup normal in $G / G^{\prime \prime}$ and having an index relatively prime to its order, unless it is a $p$-group. In the former case $G$ splits over this normal subgroup since it is a Hall subgroup. Therefore $G / G^{\prime \prime}$ is a $p$-group. But $\left(G / G^{\prime \prime}\right) / \Phi\left(G / G^{\prime \prime}\right)=T$ must be inseparable. Hence $T$ is cyclic of prime order. This contradicts $G^{\prime} \neq G^{\prime \prime}$. Hence $G^{\prime}=G^{\prime \prime}$ and so $G$ is abelian. The converse is evident.

Next, consider (2). Let $N$ be a minimal normal subgroup of $G$. By (1), $G / N$ is cyclic of prime power order. If $G$ is a $p$-group, then $|N|=p$. Hence $G$ is of type (a). Suppose that $(|G / N|,|N|)=1$. Then $G$ satisfies (b) whenever $G$ is abelian. If $G$ is nonabelian, then $N$ coincides with its centralizer in $G$. So $G$ has form (c). Again, the converse is evident.

If the condition of solvability is relaxed in Theorem 2.2, then the resulting groups are not sharply defined. For example, the special linear groups $\operatorname{SL}(2, q)$ for primes $q>3$ would be included in the classification of (1) and (2) would include the symmetric groups of degree $n \geqslant 5$ as well as each direct product of two nonabelian simple groups.

Next let us examine the role of the $E$-residual in an inseparable group.

THEOREM 2.3. A group $G=[N] S$ if and only if there exists a reduced product $G=N S$ such that $G_{E}=\left[N \cap G_{E}\right] S_{E}$.

Proof. Let $G=[N] S$. Since $E$ is a subgroup inherited formation, then $H_{E} \subseteq G_{E}$ for all subgroups $H$ in $G$. In particular, $S_{E} \subseteq G_{E}$. Moreover $G / N S_{E}$ $\in E$ implies that $G_{E} \subseteq N S_{E}$. Hence $G_{E}=G_{E} \cap N S_{E}=S_{E}\left(N \cap G_{E}\right)=$ $\left[N \cap G_{E}\right] S_{E}$.

Suppose that $G=N S$ is a reduced product for $N \triangleleft G$ such that $G_{E}=$ $\left[N \cap G_{E}\right] S_{E}$. Then $N \cap S \subseteq \Phi(S) \subseteq S_{E}$ implies that $N \cap S \subseteq\left(N \cap G_{E}\right) \cap S_{E}$ $=1$. Therefore $G=[N] S$.

In general, if $G=N T$ for $N \triangleleft G$, then $G_{E}=\left(N \cap G_{E}\right) T_{E}$.

COROLLARY 2.3. If $G_{E}$ is inseparable, then $G=[N] S$ if and only if $N \cap G_{E}$ $=1$ or there exists a reduced product $N T$, for $T \subseteq S$, such that $T \in E$.

Proof. The only part of the proof that needs to be mentioned is that if $G=N S$ yields a reduced product $G=N T, T \subseteq S$, and $T \in E$, then $N \cap T \subseteq$ $\Phi(T)=1$ implies that $G=[N] T$. 
THEOREM 2.4. A solvable group $G$ is inseparable if and only if

(1) $G_{E} \neq\left[N \cap G_{E}\right] S_{E}$ whenever $G=N S$ is a reduced product for each proper normal subgroup $N$ and $1<\left|N \cap G_{E}\right|<\left|G_{E}\right|$,

(2) the normal subgroup $\Omega(G)$ generated by all the elementary abelian $p$ groups for all $p$ dividing $|G|$ is contained in the intersection $R$ of all normal maximal subgroups of $G$, and

(3) each minimal normal subgroup of $G$ is in $\Phi(G)$.

Proof. Let $G$ be inseparable. Then (1) is valid by Theorem 2.3, and clearly $\Omega(G) \subseteq R$. Let $M$ be a minimal normal subgroup of $G$. Then $M \cap \Phi(G)$ $=1$ implies that $G$ splits over $M$. Hence (3) is necessary.

Suppose that conditions (1)-(3) hold and that $G=[N] T$ for $N \neq 1 \neq T$. By Theorem 2.3, $G_{E}=\left[N \cap G_{E}\right] T_{E}$. By (1), $N \cap G_{E}=1$ or $G_{E}$. If $N \cap G_{E}$ $=1$, then $N$ contains a minimal normal subgroup of $G$ that is not contained in $\Phi(G)$. This contradicts (3). Therefore $G_{E} \subseteq N$ and $T \in E$. By (2), $T \subseteq \Omega(G)$ $\subseteq R$. Since $N$ is contained in a normal maximal subgroup $M$ of $G$, then $N T \subseteq$ $\bar{M} \subseteq G$. This contradiction implies that $G$ is inseparable.

COROLlaRY 2.4. A p-group $G$ is inseparable if and only if

(1) $\Phi(G) \neq[N \cap \Phi(G)] \Phi(S)$ whenever $G=N S$ is a reduced product for each proper normal subgroup $N$ and $1<|N \cap \Phi(G)|<|\Phi(G)|$, and

(2) $\Omega_{1}(G) \subseteq \Phi(G)$.

3. The $E$-residual as a $p$-group; general comments. If $G_{E}$ is a $p$-group, then a Sylow $p$-subgroup $P$ of $G$ has a nontrivial Frattini subgroup. All Sylow $q$-subgroups for $q \neq p$ are elementary abelian. As a result of the next theorem, $G_{F}=$ $\Phi(G)$.

THEOREM 3.1. For a solvable group $G, G_{F}$ is nilpotent if and only if $G_{F}$ $=\Phi(G)$.

Proof. From [6] it is known that the identity element is the prefrattini subgroup of a solvable group $G$ if and only if each normal subgroup in $G$ possesses a complement. Hence $G_{F}$ is the least normal subgroup in $G$ generated by the prefrattini subgroups in $G$. Since $\Phi(G) \subseteq W$ for a prefrattini subgroup $W$ in $G$, then $W / \Phi(G)$ is a prefrattini subgroup of $G / \Phi(G)$ (see [6]). Moreover, $(G / \Phi(G))_{F}$ $=G_{F} / \Phi(G)=\left\langle W^{g} \Phi(G) \mid g \in G\right\rangle$. If $G_{F} / \Phi(G) \neq \Phi(G)$, then $G_{F} / \Phi(G) \subseteq F(G) / \Phi(G)$ is avoided by $W / \Phi(G)$ since a prefrattini subgroup avoids all complemented chief factors. The contradiction implies that $G_{F}=\Phi(G)$. The converse is evident.

COROLLARY 3.1. If $G_{E}$ is a p-group and $G$ is solvable inseparable, then $G_{F}=\Phi(G) \neq 1$.

Condition (2) in Theorem 2.4 yields the next result. 
(3.2) If $G$ is solvable inseparable and $G_{E}$ is a $p$-group, then each normal maximal subgroup of $G$ has index $p$ in $G$.

THEOREM 3.3. Let $G$ be an inseparable nonnilpotent solvable group, $G_{E} a$ p-group, and $P$ a Sylow p-subgroup of $G$. The following properties are satisfied:

(1) $\Phi(G) \subset G_{E}$.

(2) $\Phi(P) \not G$.

(3) $G_{E}$ cannot be a cyclic group of prime power order.

(4) $\left(G_{E}: \Phi(G)\right) \geqslant p^{2}$.

(5) $G_{E}=F(G), F(G)$ the Fitting subgroup of $G$, whenever $F(G)$ is metacyclic.

(6) If $\left(G_{E}: \Phi(G)\right)=p^{2}$, then $G_{E} / \Phi(G)$ is an irreducible subgroup in $G / \Phi(G)$.

Proof. If $G$ is inseparable, then $F(G)$ is a $p$-group. Otherwise $G$ would split over each nontrivial direct $q$-factor of $F(G)$ for $q \neq p$. Suppose that $\Phi(G)=$ $G_{E}$. Then $P / \Phi(G)$ is abelian for each Sylow $p$-subgroup $P$. Since $\Phi(G) \subset F(G)$ in a solvable group $G$ and $C_{G}(F(G) / \Phi(G)) \subseteq F(G) / \Phi(G)$, then $P / \Phi(G) \subseteq F(G) / \Phi(G)$. Therefore $P \triangleleft G$ and $G$ splits over $P$. This contradicts the inseparability of $G$; (1) is valid.

By (1.3), $\Phi(P)=G_{E}$ whenever $\Phi(P) \triangleleft G$. Since $\Phi(P) \triangleleft G$ implies that $\Phi(P) \subseteq \Phi(G)$, then $\Phi(G)=G_{E}$. This contradicts (1) and so verifies (2).

(3) is an immediate consequence of (2).

For (4), suppose that $\left(G_{E}: \Phi(G)\right)=p$. Then $\Phi(P / \Phi(G)) \triangleleft G / \Phi(G)$. Hence $\Phi(P / \Phi(G))=\Phi(G)$. So $\Phi(P) \subseteq \Phi(G)$. This implies $G_{E}=\Phi(G)$, contradicting (1).

Since $\Phi(F(G)) \subseteq \Phi(G) \subset G_{E} \subseteq F(G)$, then $F(G)=G_{E}$ by (4). This verifies (5).

Examine (6). $G_{E} / \Phi(G)$ is completely reducible. If $G_{E} / \Phi(G)$ is the direct product of two subgroups of order $p$ and normal in $\bar{G}=G / \Phi(G)$, then at least one of them, say $\bar{A}$, is noncentral in $\bar{G}$. Hence $p \nmid\left(\bar{G}: C_{\bar{G}}(\bar{A})\right)$. But this implies that $G$ contains a normal maximal subgroup of index other than $p$. (3.2) is contradicted.

Theorem 3.3(5) motivates the remainder of the article. If $G_{E}$ is a $p$-group and $G$ is inseparable, then $G_{E}$ cannot be cyclic by Theorem 3.3(3). Because of Example 1.2, it seems reasonable to restrict $G_{E}$ to being a noncyclic metacyclic $p$-group. As the next theorem points out, the class of nonnilpotent solvable groups minimal with the property of being inseparable and having $G_{E}$ a metacyclic $p$ group will also have $F(G)=G_{E}$. Therefore the condition put on $F(G)$ in Theorem 3.3(5) arises in a nontrivial way. In $\S 5$, it will be shown that this class reduces to the group of Example 1.2.

LEMMA 3.4. Let $G$ be an inseparable nonnilpotent solvable group such that $G_{E}$ is a metacyclic p-group. Suppose that $G / \Phi(G)=[N / \Phi(G)](A / \Phi(G)), N \cap G_{E}$ 
$\subseteq \Phi(G)$, and $A=[B] C$ for $B, C \neq 1$. Then

(1) $G_{E}=A_{E}=C_{E}$ and

(2) $G / \Phi(G)=[N B / \Phi(G)](C / \Phi(G))$ with $N B \cap G_{E} \subseteq \Phi(G), B \not \subset N, C \not \subset N B$.

Proof. By Theorem 2.3, $A_{E}=\left[B \cap A_{E}\right] C_{E}$. Moreover $A_{E} \subseteq G_{E} \cdot G_{E}$ metacyclic implies that $\Phi\left(G_{E}\right)=\Phi(G)$, by Theorem 3.3(4). Therefore $G_{E}=$ $G_{E} \cap N A_{E}=A_{E}\left(N \cap G_{E}\right) \subseteq A_{E} \Phi(G)=A_{E} \Phi\left(G_{E}\right)=A_{E}$.

Suppose $B \cap A_{E} \nsubseteq \Phi(G)$. ( $\left(B \cap A_{E}\right) \triangleleft A$ and $\left[N, G_{E}\right] \subseteq \Phi(G)$. Hence $\Phi(G)\left(B \cap A_{E}\right) \triangleleft G$. By Theorem 3.3(6), $G_{E} / \Phi(G)$ is irreducible in $G / \Phi(G)$. Since $B \cap A_{E} \nsubseteq \Phi(G)$, then $\Phi(G)\left(B \cap A_{E}\right)=A_{E}=\Phi\left(A_{E}\right)\left(B \cap A_{E}\right)=\left(B \cap A_{E}\right)$. Since $B \cap C=1$, then $C_{E}=1$. This implies that $A=[B] C$ and $C \in E$. Since $N B \triangleleft G$, then $G=[N B] C^{*}$ for some subgroup $C^{*} \subseteq C$. But $G$ is inseparable. Hence $G=N B$. Therefore $A=N B \cap A=B(N \cap \bar{A})=B \Phi(G)=B \Phi\left(A_{E}\right)=B$. This contradicts $C \neq 1$ in the hypothesis. Therefore $\left(B \cap A_{E}\right) \subseteq \Phi(G)=\Phi\left(A_{E}\right)$. So $A_{E}=\left[B \cap A_{E}\right] C_{E}=C_{E}$.

Since $\Phi(G) \subset C_{E}$, consider $G=(N B / \Phi(G))(C / \Phi(G))$. Let $x \in N B \cap C$. Then $x=c=n b$, for $c \in C, n \in N$, and $b \in B$, and $n=c b^{-1} \in N \cap A \subseteq \Phi(G)$. Therefore $n \in C$. This implies that $b=1$ and $x=n \in \Phi(G)$. So $N B \cap C \subseteq$ $\Phi(G)$. Consequently $G / \Phi(G)=[N B / \Phi(G)](C / \Phi(G))$. Moreover since $G_{E}=C_{E}$, then $N B \cap G_{E} \subseteq \Phi(G)$.

THEOREM 3.5. Let $G$ be minimal as an inseparable nonnilpotent solvable group having $G_{E}$ as a metacyclic p-group. Then $F(G)=G_{E}$.

Proof. Suppose that $G_{E} \neq F(G)$. Since $G_{E} / \Phi(G)$ is irreducible in $G / \Phi(G)$ and $F(G) / \Phi(G)$ is completely reducible, there exists an irreducible factor $M / \Phi(G)$ $\subset F(G) / \Phi(G)$ such that $M \cap G_{E} \subseteq \Phi(G)$. Hence $G / \Phi(G)=[M / \Phi(G)]\left(A^{*} / \Phi(G)\right)$ and $M \cap A^{*}=\Phi(G)$. Let $N$ be a normal subgroup maximal with respect to the property that $M \subseteq N, G / \Phi(G)=[N / \Phi(G)](A / \Phi(G)), N \cap G_{E} \subseteq \Phi(G)$, and $N$ is proper in $G$. By Lemma 3.4, $A_{E}=G_{E}$. Suppose that $A$ is inseparable. Then the minimality of $G$ is contradicted unless $A$ is nilpotent. So $A$ would be a $p$-group. Therefore $A_{E}=\Phi(A)$. Since $\Phi(G) \neq 1$, then $\Phi(A) \neq 1$. Since $\Phi(A) \subseteq \Phi(P) \subseteq$ $G_{E}$ for a Sylow $p$-subgroup $P$ of $G$ that contains $A$, it follows that $\Phi(P) \triangleleft G$. This contradicts Theorem 3.3(2). Therefore $A$ is separable, say $A=[B] C$ with $B \neq 1$ and $C \neq 1$. By Lemma $3.4, G / \Phi(G)=[N B / \Phi(G)](C / \Phi(G))$ and $N B \cap G_{E} \subseteq \Phi(G)$. A contradiction is reached in that $N$ is not maximal with respect to the required properties. This contradiction arose by having assumed that $G_{E} \neq F(G)$.

4. $p$-groups with a metacyclic Frattini subgroup. For $p$-groups, $G_{E}=\Phi(G)$. Since Berkovič [4] has already identified the structure of these groups, then his results will be used in both this section and the next in order to characterize the inseparable group G. A summary from [4] is given in (4.1). 
A $p$-group $P$ is absolutely regular if $\left(P: \mho_{1}(P)\right)<p^{p}$. Each absolutely regular $p$-group is regular and an absolutely regular 2-group is cyclic. If $\Phi(P)$ is metacyclic, then $P$ has the following structure:

(4.1) For $\Phi(P)$ cyclic, $P=A B$ such that $A$ is generated by $\Phi_{0}=\Omega_{1}(\Phi(P))$ and all normal subgroups of $P$ containing $\Phi_{0}$ of type $(p, p), B$ is either cyclic or a 2-group of maximal class, $\Phi(P) \subseteq B$, and $A / \Phi_{0} \subseteq Z\left(P / \Phi_{0}\right)$. For $\Phi(P)$ noncyclic, $A$ is generated by $\Phi_{0}=\Omega_{1}(\Phi(P))$ and all normal subgroups of $P$ having order $p^{3}$, exponent $p$, and containing $\Phi_{0}, B$ is absolutely regular or a 3-group of maximal class, $\Phi(P) \subseteq B$, and $A / \Phi_{0}$ is elementary abelian and contained in $Z\left(P / \Phi_{0}\right)$.

THEOREM 4.2. Let $G$ be an inseparable p-group with a cyclic Frattini subgroup. Then $G$ is either cyclic or a generalized quaternion group.

Proof. Since $G$ is inseparable, then $\Omega_{1}(G)=\Omega_{1}(\Phi(G))$ by Corollary 2.4. By (4.1), $A=\Phi_{0}$ and $G=B$. The 2-groups of maximal class are known and of these only the generalized quaternion group is inseparable.

THEOREM 4.3. Let $G$ be an inseparable p-group and $\Phi(G)$ be metacyclic, but not cyclic, for $p>2$. Then $G$ is either metacyclic or of maximal class. If $G$ is of maximal class, then $p=3$.

Proof. Since $G$ is inseparable, then $A=\Phi_{0}$ and $G=B$, by (4.1). If $G$ is regular, then $\left|\Phi_{0}\right|=p^{2}$ implies that $G$ is metacyclic (see p. 337 of [8]).

The corollary to Theorem 4.8 will remove the possibility in Theorem 4.3 that an inseparable 3-group of maximal class could be metacyclic. However, other inseparable 3-groups of maximal class exist that satisfy the conclusion of Theorem 4.3, for example, consider

$$
G=\left\langle a, b, c \mid a^{9}=b^{3}=c^{9}=1, a b=b a, c^{-1} a c=a b, c^{-1} b c=b a^{3}, c^{3}=a^{3}\right\rangle .
$$

$G$ is neither metacyclic nor regular.

More generally, the best possible result about inseparable $p$-groups of maximal class seems to be the following:

(4.4) A $p$-group $G$ of maximal class is inseparable if and only if $\Omega_{1}(G)=$ $\Omega_{1}(\Phi(G))$.

For $p>2$, the characterization of a nonabelian inseparable metacyclic $p$ group can be stated explicitly. By Theorem 4.2, we know that $\Phi(G)$ cannot be cyclic. As is known a metacyclic $p$-group $G$ has the form

(4.5) $G=\left\langle a, b \mid a^{p^{n}}=1, b^{p^{m}}=a^{p^{t}}, b^{-1} a b=a^{k}\right\rangle$ with $t \geqslant 0, k^{p^{m}}-1 \equiv$ $p^{t}(k-1) \equiv 0 \bmod p^{n}$, and $|G|=p^{n+m}$.

LEMMA 4.6. Let $G$ be a nonabelian p-group in (4.5) for $p>2$. Then $G$ splits over $\langle a\rangle$ if and only if $\exp (G)=p^{n}$ or $p^{m}$.

Proof. Since $\mho_{i}(G)=\Phi^{i}(G)=\Phi\left(\Phi^{i-1}(G)\right)=\left\langle a^{p^{i}}\right\rangle\left\langle b^{p^{i}}\right\rangle$, for $\Phi^{0}(G)=G$, 
$\Phi^{1}(G)=\Phi(G)$, and $|G|=p^{n+m}$, then $G=[\langle a\rangle]\langle b\rangle$ implies that $\exp (G)=$ $\exp (\langle a\rangle)$ or $\exp (\langle b\rangle)$.

Now suppose that $\exp (G)=p^{n}$ or $p^{m}$. Clearly if $\exp (G)=p^{m}$, then $G$ splits over $\langle a\rangle$. Let $\exp (G)=p^{n}$. Then $\exp (\langle b\rangle)=p^{n+m-t} \leqslant p^{n}$. Therefore $m \leqslant t$. So $\Phi^{m-1}(G)=\left\langle a^{p^{m-1}}\right\rangle\left\langle b^{p^{m-1}}\right\rangle$ is noncyclic, $\Phi^{m}(G)=\left\langle a^{p^{m}}\right\rangle$, and $\left\langle a^{p^{m-1}}\right\rangle$ is a maximal subgroup of $\Phi^{m-1}(G)$. Since $\Phi^{m-1}(G)$ is not cyclic, then $\left|\Omega_{1}\left(\Phi^{m-1}(G)\right)\right|=p^{2}$. There exists an element $x \in \Phi^{m-1}(G)$ such that $\Phi^{m-1}(G)$ $=\left[\left\langle a^{p^{m-1}}\right\rangle\right]\langle x\rangle$. From the regularity of $G$, this implies that there exists $y \in G$ such that $G=[\langle a\rangle]\langle y\rangle$ and $y^{p^{m-1}}=x$. Therefore $G$ splits over $\langle a\rangle$.

LEMMA 4.7. Let $G$ be an inseparable nonabelian p-group for $p>2$ given by (4.5). Then

(1) $\langle b\rangle \pitchfork G$ and

(2) $\exp (G)=p^{n+m-t}$ for $0<t<n, m>t$, and $0<(k-1)<p^{t}<p^{n}$.

Proof. Since $G$ is nonabelian and inseparable, then $0<t<n$. By Lemma 4.6, $\exp (G) \neq p^{n}$ nor $p^{m}$. Therefore $\exp (G)=p^{m+n-t}=\exp (\langle b\rangle)>p^{n}$. Hence $m>t$. If $\langle b\rangle \triangleleft G$, then $\exp (G)=\exp (\langle b\rangle)$ by application of Lemma 4.6 to $\langle b\rangle$. Hence $\langle b\rangle \not G$. If $(k-1) \geqslant p^{t}$, then $\langle b\rangle \triangleleft G$. So $0<(k-1)<p^{t}$.

THEOREM 4.8. Let $G$ be a nonabelian metacyclic p-group for $p>2$ as given in (4.5). $G$ is inseparable if and only if $\exp (G)=p^{m+n-t}$, for $0<t<n$, $m>t$, and $0<(k-1)<p^{t}<p^{n}$.

Proof. Lemma 4.7(2) states the necessity.

Suppose that $G$ satisfies the conditions. First note that

$$
G / G^{\prime}=G /\left\langle a^{k-1}\right\rangle=\left(\langle a\rangle /\left\langle a^{k-1}\right\rangle\right)\left(\left\langle a^{k-1}\right\rangle\langle b\rangle\left\langle\left\langle a^{k-1}\right\rangle\right)\right.
$$

and

$$
\langle a\rangle \cap\left\langle a^{k-1}\right\rangle\langle b\rangle=\left\langle a^{k-1}\right\rangle\left\langle a^{p^{t}}\right\rangle=\left\langle a^{k-1}\right\rangle .
$$

Hence $G /\left\langle a^{k-1}\right\rangle$ is abelian of type $\left(k-1, p^{m}\right)$ since $\mid\left\langle a^{k-1}\right\rangle\langle b\rangle\left\langle\left\langle a^{k-1}\right\rangle\right|=p^{m}$. Assume that $G=[N] A$ and $1<|N|<|G|$. Since $G$ is nonabelian and $p>2$, then $\left|\Omega_{1}(G)\right|=p^{2}$. Hence $N$ and $A$ are cyclic; $G^{\prime} \subset N$. Moreover $G / G^{\prime}=$ $N /\left\langle a^{k-1}\right\rangle \otimes\left\langle a^{k-1}\right\rangle A /\left\langle a^{k-1}\right\rangle$ has order $p^{n+m} /(k-1)$. Then $N /\left\langle a^{k-1}\right\rangle$ has order $(k-1)$ or $p^{m}$. If $\left|N /\left\langle a^{k-1}\right\rangle\right|=(k-1)$, then $|N|=p^{n}$ and $|A|=p^{m}$. Hence $\exp (G)=p^{n}$ or $p^{m}$ which contradicts the hypothesis. If $\left|N /\left\langle a^{k-1}\right\rangle\right|=p^{m}$, then $|N|=p^{n+m} /(k-1)$ and $|A|=(k-1)$. Since $\exp (G) \neq(k-1)$, then $\exp (G)=$ $p^{n+m} /(k-1)>p^{n+m-t}$. This contradicts the hypothesis. Therefore $G$ is inseparable.

COROLlary 4.8. An inseparable 3-group $G$ of maximal class having $\Phi(G)$ metacyclic, but not cyclic, cannot be metacyclic. 
Proof. Let $G$ be a metacyclic 3-group satisfying the hypothesis and having defining relations (4.5). The class of $G$ is $n=n+m-2$. Hence $m=2$. By Theorem 4.8, $t=1$. Since $(k-1) \geqslant 3$, a contradiction is reached.

The class of groups satisfying Theorem 4.8 is certainly not empty. Just consider $G=\left\langle a, b \mid a^{p^{4}}=1, b^{p^{4}}=a^{p^{3}}, b^{-1} a b=a^{1+p^{2}}, p \neq 2\right\rangle$ of order $p^{8}$ and exponent $p^{5}$.

Even though Theorem 4.8 identifies succinctly the inseparable metacyclic $p$-groups for $p>2$, it also points out that all other types can be expressed as splitting extensions.

Nothing further will be discussed for the prime $p=2$, since for other than the cases presented by 0 . Tausky (see p. 339 of [8]), there does not seem to be a well-defined direction in which to go toward the identification of the 2-groups having a noncyclic metacyclic Frattini subgroup. Of the metacyclic 2-groups of maximal class, only the generalized quaternion group is inseparable.

5. Minimal inseparable nonnilpotent solvable groups with the $E$-residual a metacyclic $p$-group. In view of Theorem 3.5, the groups $G$ to be considered in this section will be nonnilpotent and solvable having $F(G)$ metacyclic and $G_{E}$ a noncyclic metacyclic $p$-group. The main result of this section is the following:

THEOREM 5.1. Let $G$ be a nonnilpotent solvable group having a metacyclic Fitting subgroup. If $G_{E}$ is a p-group, then $G$ is separable whenever $G$ is not the group of Example 1.2.

The proof will be a culmination of a succession of lemmas. Let $G$ be an inseparable nonnilpotent solvable group with $G_{E}=F(G)$ a noncyclic metacyclic $p$-group and denote a Sylow $p$-subgroup by $P$. The following properties are immediate consequences of the properties of the Frattini subgroup.

(5.2) $\Phi\left(G_{E}\right) \subseteq \Phi(G) \cap \Phi(P)$.

(5.3) $\Phi(G)=\Phi\left(G_{E}\right)$.

(5.4) $\left(G_{E}: \Phi(G)\right)=p^{2}$ and $G_{E} / \Phi(G)$ is irreducible in $G / \Phi(G)$.

(5.5) $\Phi(G) \subset \Phi(P) \subset G_{E}$.

LEMMA 5.6. Let $G$ be a nonnilpotent solvable group. If $F(G)$ is metacyclic, $G_{E}$ is a metacyclic p-group for $p>2$, and $\Phi(G)$ is noncyclic, then $G$ is separable.

Proof. Assume that $G$ is inseparable and let $P$ denote a Sylow $p$-subgroup of $G$. By (5.5), $\Phi(P)$ is noncyclic. In the notation of (4.1), let $P=A B$. Since $\Phi(G)$ is noncyclic, then $\Phi_{0} \subseteq \Phi(G)$. Hence $P / \Phi(G)=(A \Phi(G) / \Phi(G))(B / \Phi(G))$. By (4.1), $A \Phi(G) / \Phi(G)$ centralizes $F(G) / \Phi(G)$. Hence $A \subseteq A \Phi(G) \subseteq F(G)=G_{E}$. $A$ is metacyclic and cannot contain subgroups of order $p^{\overline{3}}$ having exponent $p$. So $A=\Phi_{0} \subseteq \Phi(P)$. Therefore $P=A B=B$ and $P / \Phi(G)$ is a nonabelian $p$-group of 
order $p^{3}$. Since $\Phi_{0}=\Omega_{1}(P)$ has order $p^{2}$, then if $P$ is regular, $P$ is metacyclic (see p. 337 of [8]). Consequently $G_{E} / \Phi(G)$ is the unique elementary abelian subgroup of $P / \Phi(G)$. But $G_{F}=\Phi(G)$ by Theorem 3.1. Since $G$ is inseparable, then each normal maximal subgroup $M$ of $G$ has index $p$. However $G / \Phi(G)$ splitting over $M / \Phi(G)$ implies that $P / \Phi(G)$ splits over $G_{E} / \Phi(G)$. This contradicts $\Omega_{1}(P / \Phi(G))=G_{E} / \Phi(P)$. Hence $P$ cannot be regular. So assume $P$ to be of maximal class. Then $p=3$ and $G / G_{E}$ is isomorphic to a subgroup of $G L(2,3)$. But $G L(2,3)$ contains no subgroups that have all Sylow subgroups elementary abelian and in which the normal maximal subgroups have only index 3 . So $P$ cannot have maximal class. Hence $G$ is separable.

LEMmA 5.7. Let $G$ be a nonnilpotent solvable group. If $F(G)$ is metacyclic, $G_{E}$ is a metacyclic 2-group, and $\Phi(G)$ is noncyclic, then $G$ is separable.

Proof. Assume that $G$ is inseparable. Then $G_{E}=F(G), \dot{\Phi}(G)=\Phi\left(G_{E}\right)$, $G_{E} / \Phi(G)$ is an irreducible subgroup in $G / \Phi(G)$, and $G / G_{E}$ is isomorphic to the symmetric group of degree three. Since $\Phi\left(G_{E}\right)$ is noncyclic, there is a smallest integer $k$ such that $\Phi^{k}\left(G_{E}\right)$ is noncyclic and $\Phi^{k+1}\left(G_{E}\right)$ is cyclic. $\Omega_{1}\left(G_{E}\right) \subset$ $\Phi^{k}\left(G_{E}\right)$. Let $\alpha$ denote an automorphism of order three induced by $G$ on $G_{E}$. If $\Phi^{k+1}\left(G_{E}\right) \neq 1$, then $A=\Phi^{k}\left(G_{E}\right) / \Phi^{k+2}\left(G_{E}\right)$ has order $2^{3}$. $A$ cannot be nonabelian (see p. 306 of [8]). Hence $A$ is abelian of type (2.1). Therefore $\alpha$ acts trivially on $A$ and hence on $\Phi^{k}\left(G_{E}\right) / \Phi^{k+1}\left(G_{E}\right)$.

Next consider $G_{E}^{*}=G_{E} / \Phi^{2}(G)$ and $\Phi\left(G_{E}^{*}\right)=\Phi(G) / \Phi^{2}(G)$. Since $\Phi\left(G_{E}^{*}\right)$ is elementary abelian and $G_{E}^{*}$ is metacyclic, then $\Phi\left(G_{E}^{*}\right) \subseteq Z\left(G_{E}^{*}\right) . \Phi\left(G_{E}^{*}\right)$ contains all the proper $\alpha$-invariant subgroups of $G_{E}^{*}$. Hence $G_{E}^{*}$ is abelian of type $\left(2^{2}, 2^{2}\right)$. This implies that $\Phi\left(G_{E}^{*}\right)$ is irreducible under $\alpha$. By an induction argument, $\Phi^{j}\left(G_{E}\right) / \Phi^{j+1}\left(G_{E}\right)$ is irreducible under $\alpha$ for $1 \leqslant j \leqslant k$. If $\Phi^{k+1}\left(G_{E}\right) \neq 1$, there is a contradiction. Therefore $\Phi^{k+1}\left(G_{E}\right)=1$ and $\Phi^{k}\left(G_{E}\right)=\Omega_{1}\left(G_{E}\right)$.

Since $\Phi(G) \neq G_{E}$, there exists a reduced product $G=G_{E} A$ such that $G_{E} \cap A$ $=\Phi(A)=A_{E}$ and $A / G_{E} \cap A$ is isomorphic to the symmetric group of degree 3. $\Phi(A)$ is cyclic, for otherwise $A / \Phi^{2}(A)$ is a group of order 24; such groups have $\left|\Phi(A) / \Phi^{2}(A)\right| \leqslant 2$. Since $\Phi(A)=A_{E}$, then $\Phi(P)=\Phi(A)$, by (1.3), for a Sylow 2 -subgroup $P$ of $A$. Hence $P=\langle b\rangle$ is cyclic. The inseparability of $G$ implies that $b^{2} \neq 1$. For some integer $t,\left\langle b^{2} t\right\rangle$ is a nontrivial subgroup of $\Omega_{1}\left(G_{E}\right)$. This leads to a contradiction since $\Omega_{1}\left(G_{E}\right)$ is $A$-irreducible, and yet $A$ centralizes $\left\langle b^{2}{ }^{t}\right\rangle$. Hence $G$ is separable.

LEMMA 5.8. Let $G$ be a nonnilpotent solvable group. If $F(G)$ is metacyclic, $G_{E}$ is a metacyclic p-group, $P$ is a Sylow p-subgroup of $G$ having $\Phi(P)$ noncyclic, and $\Phi(G)$ is cyclic, then $G$ is separable.

Proof. Assume $G$ to be inseparable. Since $G_{E}$ and $\Phi(P)$ are noncyclic, then 
$\Omega_{1}\left(G_{E}\right)=\Omega_{1}(\Phi(P)) \triangleleft G$. Moreover $\Phi(P)=\Phi(G) \Omega_{1}(\Phi(P))$. Hence $\Phi(P) \triangleleft G$. This contradicts Theorem 3.3(2).

LEMMA 5.9. Let $G$ be an inseparable nonnilpotent solvable group and $F(G)=G_{E}$ be metacyclic. Then $G_{E}$ is a generalized quaternion group if and only if $G$ is the group of Example 1.2.

Proof. Let $P$ denote a Sylow 2-subgroup of $G$ and suppose that $G_{E}$ is a generalized quaternion group of order $2^{n}$. If $n \geqslant 4$, then $G_{E}$ has a unique cyclic maximal subgroup $M \triangleleft G$. Since $\Phi(P)$ cannot be nonabelian with a cyclic center (see p. 306 of [8]) and $\Phi(P) \pitchfork G$ by Theorem 3.3(2), then $\Phi(P) \nsubseteq M$ and $|\Phi(P)|$ $=4$. But $\left|\Phi\left(G_{E}\right)\right|=2^{n-2}$ and $\Phi\left(G_{E}\right) \subseteq \Phi(P)$. Therefore $\Phi\left(G_{E}\right)=\Phi(P)$ which implies that $\Phi(P) \triangleleft G$. So $n=3$ and $G_{E}$ is the quaternion group. Since $\Phi(G)$ $\neq G_{E}$ and $\Phi(G) \neq 1$, then $\mid \Phi(G \mid=2$ or 4. By Theorem 3.3(4), $|\Phi(G)|=2$. Since $\Phi(P) \not G$, then $\Phi(P)$ has order 4. $G_{E}=F(G)$ implies that $C_{G}(F(G))=$ $Z\left(G_{E}\right)=\Phi(G)$. Therefore $G / \Phi(G)$ is isomorphic to a subgroup of the symmetric group $S_{4}$ of degree four that contains the normal Klein four-group. This subgroup cannot have order a power of two nor can it just contain the alternating group. Hence $G / \Phi(G) \cong S_{4}$. The subgroup $H=\left[G_{E}\right] T, T$ a Sylow 3-subgroup of $G$, is normal in $G$. Since $G=H N_{G}(T)$, there exists an element $y \in N_{G}(T)$ of exponent $\left.2^{m}, m\right\rangle 1$. $\langle y\rangle$ cannot centralize $T$ and $\langle y\rangle \Phi(G) / \Phi(G)$ must have order two and normalize a Sylow 3-subgroup of $G / \Phi(G)$. Therefore $\left\langle y^{2}\right\rangle=\Phi(G)=\Phi\left(G_{E}\right)$. Hence there exists a subgroup $K=[T]\langle y\rangle$ such that the homomorphism $\theta:\langle y\rangle$ $\rightarrow \operatorname{Aut}(T)$ has kernel $\left\langle y^{2}\right\rangle$. Then $G=G_{E} K$ such that $G_{E} \cap K=Z\left(G_{E}\right)=\left\langle y^{2}\right\rangle$, $G /\left\langle y^{2}\right\rangle \cong S_{4}$, and $K /\left\langle y^{2}\right\rangle$ is isomorphic to the symmetric group of degree three. This is the group of Example 1.2. The converse was proved in that example.

LEMMA 5.10. Let $G$ be a nonnilpotent solvable group having a metacyclic Fitting subgroup and suppose that $G_{E}$ is a p-group of maximal class. $G$ is inseparable if and only if $G$ is the group of Example 1.2.

Proof. The sufficiency has been established. First note that if $G_{E}$ is abelian, then $\left|G_{E}\right|=p^{2}$. $G_{E}$ cannot be cyclic by Theorem 3.3(3). If $G_{E}$ is elementary abelian, then $\Phi(G)=1$ by Theorem 3.3(4). Hence $G_{E}$ is not abelian.

Let $P$ be a Sylow $p$-subgroup of $G$ for $p$ dividing $\left|G_{E}\right|$. Then $\Phi(P)$ must be a maximal subgroup of $G_{E}$. Let $K_{i}(H)$ denote the $i$-term in the upper central series of the $p$-group $H=K_{1}(H)$, and $H_{1}=C_{G}\left(K_{2}(H) / K_{4}(H)\right)$. It is known that $H_{1}$ is a characteristic subgroup of index $p$ in $H$. Hence $\left(G_{E}\right)_{1}$ is a characteristic maximal subgroup of $G_{E}$ and $\left(G_{E}\right)_{1} \triangleleft G$. Assume that $\left|\left(G_{E}\right)_{1}\right| \geqslant p^{4}$. Since $K_{4}\left(G_{E}\right) \triangleleft G$, then $G / K_{4}\left(G_{E}\right)$ exists, has order equal to or greater than $p^{4}$, and $G_{E} / K_{4}\left(G_{E}\right)=\left(G / K_{4}\left(G_{E}\right)\right)_{E}$ has maximal class. Without loss of generality, assume that $K_{4}\left(G_{E}\right)=1$. Then $\left(G_{E}\right)_{1}=C_{G}\left(K_{2}\right)$ is an abelian maximal subgroup of $G_{E}$. 
$\Phi(P) \neq\left(G_{E}\right)_{1}$ by Theorem 3.3(2). If $G_{E}$ has two abelian maximal subgroups, then $\left|Z\left(G_{E}\right)\right|=p^{2}$. This contradicts $G_{E}$ having maximal class. Therefore all other maximal subgroups of $G_{E}$ are nonabelian of order $p^{3}$ and $\Phi(P)$ must be one of them. But $\Phi(P)$ cannot have a cyclic center. The contradiction arose by assuming that $\left|G_{E}\right| \geqslant p^{4}$. Hence $\left|G_{E}\right|=p^{3}$.

Since $\Phi\left(G_{E}\right) \subseteq \Phi(G)$ and $\left(G_{E}: \Phi(G)\right) \geqslant p^{2}$, then $\Phi\left(G_{E}\right)=\Phi(G)$. Suppose that $p>2$. Of the two nonabelian $p$-groups of order $p^{3}$ only one of them is metacyclic. Hence $G_{E}$ has precisely one maximal subgroup $A$ of type $(p, p)$; therefore $A \triangleleft G$. This contradicts Theorem 3.3(6). So if $G_{E}$ has maximal class, then $p=2$.

If $G_{E}$ is the dihedral group, then $G_{E}$ has a cyclic maximal subgroup that is normal in $G$. This contradicts Theorem 3.3(6). Therefore $G_{E}$ is the quaternion group and by Lemma 5.9, this is the group of Example 1.2.

LEMMA 5.11. Let $G$ be an inseparable nonnilpotent solvable group. If $F(G)=G_{E}$ is a noncyclic metacyclic p-group and $\Phi(P)$ is cyclic for a Sylow psubgroup $P$ of $G$, then $G$ is the group of Example 1.2 .

Proof. Let $p>2$. By (5.5), $1 \subset \Phi(G) \subset \Phi(P)$ and by (4.1), $P=A B$ such that $A$ is generated by $\Phi_{0}$ and all normal subgroups of type $(p, p)$ in $P$, $A / \Phi_{0} \subseteq Z /\left(P / \Phi_{0}\right), \Phi(P) \subseteq B$, and $B$ is cyclic. Hence $P / \Phi(G)$ is abelian. Since $G_{E}=F(G)$, a contradiction is reached. Therefore $p \ngtr 2$.

Let $p=2$. Then by (4.1), $P$ has the same form as above except that $B$ is either cyclic or of maximal class. The same argument just used shows that $B$ is not cyclic. Suppose that $B$ has maximal class. If $G_{E} \nsubseteq B$, then $B \cap G_{E}=\Phi(P)$. Hence $A \nsubseteq \Phi(P)$. Since $G_{E}=F(G), A \Phi(G) / \Phi(G) \subseteq G_{E} / \Phi(G)$. So $G_{E}=A \Phi(G)$. But $|\Phi(P) / \Phi(G)|=2$ implies that $B / \Phi(G) \subseteq G_{E} / \Phi(G)$. A contradiction arises since this implies that $P \subseteq G_{E}$. Therefore $G_{E} \subseteq B$ and $B=P$. Hence $G_{E}$ is a maximal subgroup of $P$ and $|P| \geqslant 2^{4}$. $P$ is either a dihedral group, a quasidihedral group, or a generalized quaternion group (see p. 339 of [8]).

If $P$ is a dihedral group, then $P$ has precisely one cyclic and two dihedral maximal subgroups. By Theorem 3.3(3), $G_{E}$ cannot be cyclic. If $G_{E}$ is a dihedral maximal subgroup, then $\Phi(P)$, being cyclic, is characteristic in $G_{E}$. Hence $\Phi(P)$ $\triangleleft G$. This contradicts Theorem 3.3(2). Consequently $G_{E}$ is not a dihedral group.

A quasidihedral group has three characteristic maximal subgroups; one is cyclic, one is generalized quaternion, and one contains $\Phi_{0}$ and has $\Phi(P)$ as a characteristic subgroup. By Theorem 3.3(3), the first case is eliminated, Lemma 5.10 settles the second case, and Theorem 3.3(2) removes the third case.

A generalized quaternion group contains one cyclic maximal subgroup and the other two maximal subgroups are generalized quaternion. Appealing to Theorem 3.3(3) and Lemma 5.10, G must be the group of Example 1.2. 
Proof of Theorem 5.1. Assume that $G$ is inseparable. $G_{E}=F(G)$ is noncyclic by Theorem $3.5(3,5)$ and $\Phi(G) \subset \Phi(P) \subset G_{E}$ for a Sylow $p$-subgroup $P$ of $G$ by (5.5). By Lemmas 5.6, 5.7, and 5.8, $\Phi(P)$ must be cyclic. Lemma 5.11 reduces this case to Example 1.2. Hence all other groups $G$ satisfying the hypothesis of Theorem 5.1 are separable.

COROLlARY 5.1. The minimal inseparable nonnilpotent solvable group with a metacyclic p-group as an E-residual is the group of Example 1.2.

Proof. By Theorem 3.5, such a group has $F(G)=G_{E}$. By Theorem 5.1, this is the group of Example 1.2.

ACKNOWLEDGMENT. I thank the Mathematics Institute at the University of Warwick for the services provided to me while this paper was being prepared.

\section{BIBLIOGRAPHY}

1. H. Bechtell, Elementary groups, Trans. Amer. Math. Soc. 114 (1965), 355-362. MR 31 \#243. $\# 1715$.

2. - The theory of groups, Addison-Wesley, Reading, Mass., 1971. MR 44

3. - On the structure of solvable nC-groups, Rend. Sem. Mat. Univ. Padova 47 (1972), 13-22. MR 48 \#2253.

4. Ja. G. Berkovix, A generalization of the theorems of Hall and Blackburn and their applications to nonregular p-groups, Izv. Akad. Nauk SSSR Ser. Mat. 35 (1971), 800-830 = Math. USSR Izv. 5 (1971), 815-844. MR 45 \#3565.

5. C. Christensen, Groups with complemented normal subgroups, J. London Math. Soc. 42 (1967), 208-216. MR 34 \#7648.

6. W. Gaschütz, Praefrattinigruppen, Arch. Math. 13 (1962), 418-426. MR 26 \#3784.

7. D. Gorenstein, Finite groups, Harper and Row, New York, 1968. MR 38 \#229.

8. B. Huppert, Endliche Gruppen. I, Die Grundlehren der math. Wissenschaften, Band 134, Springer-Verlag, Berlin and New York, 1967. MR 37 \#302.

9. H. Zassenhaus, The theory of groups, Vandenhoek and Ruprecht, Göttingen, 1956.

DEPARTMENT OF MATHEMATICS, UNIVERSITY OF NEW HAMPSHIRE, DURHAM, NEW HAMPSHIRE 03824 